\title{
Alain MASSOT
}

sociologue et professeur agrégé,

retraité du Département des fondements et pratiques en éducation, Université Laval

(2002)

\section{Une justification du revenu de citoyenneté inconditionnel et universel au dessus du seuil de pauvreté}

\section{Communication présentée au 70 Congrès de l’ACFAS, 2002.}

\author{
Un document produit en version numérique par Jean-Marie Tremblay, bénévole, \\ professeur de sociologie retraité du Cégep de Chicoutimi \\ Courriel : jean-marie tremblay@uqac.ca \\ Site web pédagogique : http ://www.uqac.ca/jmt-sociologue/ \\ Dans le cadre de : "Les classiques des sciences sociales" \\ Une bibliothèque numérique fondée et dirigée par Jean-Marie Tremblay, \\ professeur de sociologie au Cégep de Chicoutimi \\ Site web : http ://classiques.uqac.ca/
}

Une collection développée en collaboration avec la Bibliothèque Paul-Émile-Boulet de l'Université du Québec à Chicoutimi

Site web : http ://bibliotheque.uqac.ca/ 


\section{Politique d'utilisation de la bibliothèque des Classiques}

Toute reproduction et rediffusion de nos fichiers est interdite, même avec la mention de leur provenance, sans l'autorisation formelle, écrite, du fondateur des Classiques des sciences sociales, Jean-Marie Tremblay, sociologue.

Les fichiers des Classiques des sciences sociales ne peuvent sans autorisation formelle :

- être hébergés (en fichier ou page web, en totalité ou en partie) sur un serveur autre que celui des Classiques.

- servir de base de travail à un autre fichier modifié ensuite par tout autre moyen (couleur, police, mise en page, extraits, support, etc...),

Les fichiers (.html, .doc, .pdf, .rtf, .jpg, .gif) disponibles sur le site Les Classiques des sciences sociales sont la propriété des Classiques des sciences sociales, un organisme à but non lucratif composé exclusivement de bénévoles.

Ils sont disponibles pour une utilisation intellectuelle et personnelle et, en aucun cas, commerciale. Toute utilisation à des fins commerciales des fichiers sur ce site est strictement interdite et toute rediffusion est également strictement interdite.

\section{L'accès à notre travail est libre et gratuit à tous les utilisateurs. C'est notre mission.}

Jean-Marie Tremblay, sociologue

Fondateur et Président-directeur général, LES CLASSIQUES DES SCIENCES SOCIALES. 
Cette édition électronique a été réalisée par Jean-Marie Tremblay, bénévole, professeur de sociologie au Cégep de Chicoutimi à partir de :

\begin{abstract}
Alain Massot
Une justification du revenu de citoyenneté inconditionnel et universel au dessus du seuil de pauvreté.

Communication présentée au 70e Congrès de l'ACFAS, Sociologie et anthropologie. Québec : Université Laval, le 14 mai 2002, 41 pp.
\end{abstract}

[Autorisation formelle accordée par l'auteur, le 9 novembre 2012, de diffuser ce texte dans Les Classiques des sciences sociales.]

Courriel : Alain.Massot@fse.ulaval.ca

Polices de caractères utilisée : Times New Roman, 14 points.

Édition électronique réalisée avec le traitement de textes Microsoft Word 2008 pour Macintosh.

Mise en page sur papier format : LETTRE US, 8.5'” x 11'”.

Édition numérique réalisée le 25 décembre 2012 à Chicoutimi, Ville de Saguenay, Québec.

\title{
Fait avec
}

Macintosh 


\section{Alain MASSOT}

sociologue et professeur agrégé,

retraité du Département des fondements et pratiques en éducation, Université Laval

\section{Une justification du revenu de citoyenneté inconditionnel et universel au dessus du seuil de pauvreté.}

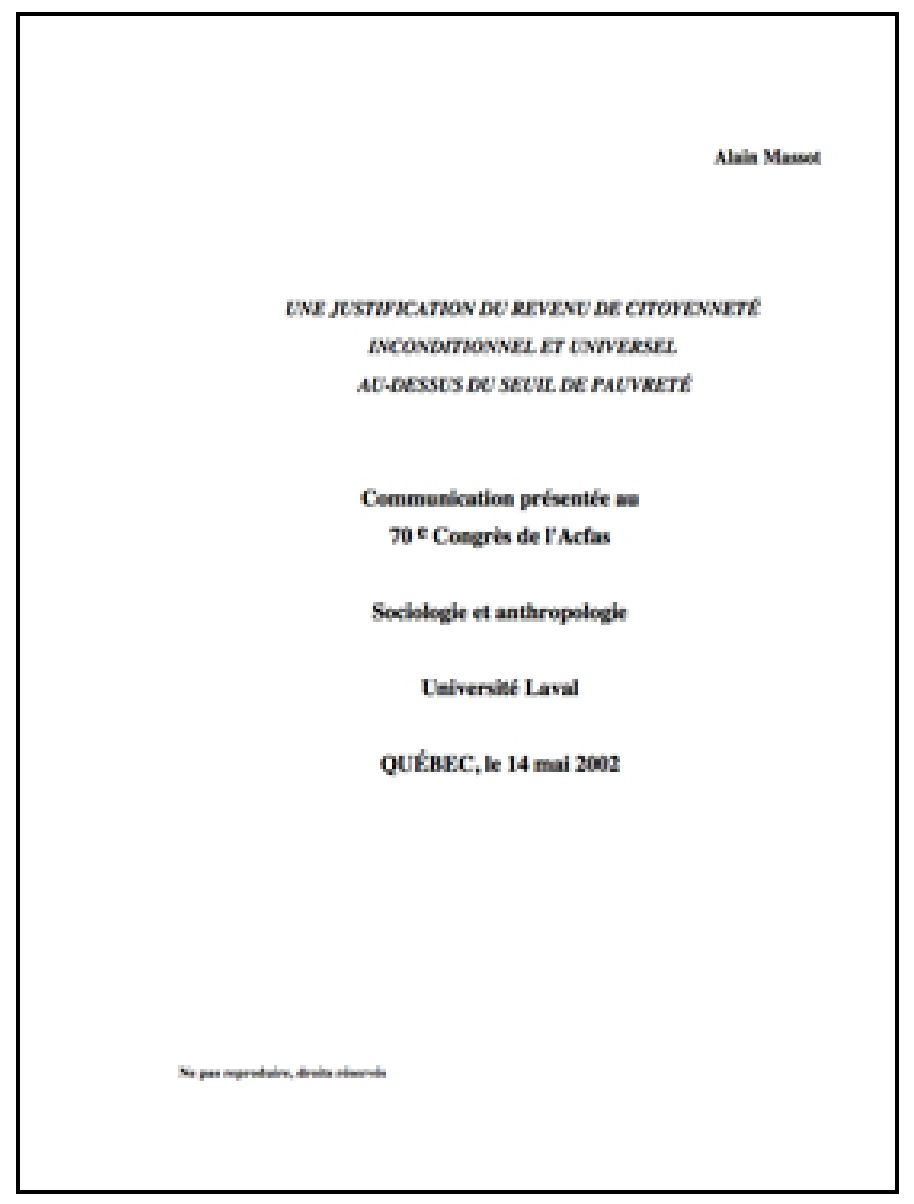

Communication présentée au 70e Congrès de l'ACFAS, Sociologie et anthropologie. Québec : Université Laval, le 14 mai 2002, 41 pp. 


\section{Table des matières}

1. Introduction [6]

2. Une justification d'ordre socio-historique [7]

2.1. La réduction du temps de travail [7]

2.2. Le taux de chômage [12]

2.3. La logique de l'investissement [16]

2.4. Le mode de production post-fordiste [18]

2.5. Le dépassement de la dualisation sociétale [21]

2.6. La révolution du temps choisi [25]

3. Un revenu de citoyenneté pour tous [28]

4. Conclusion [31]

Références [33]

ANNEXES [35]

Tableau I. Évolution des prestations d'aide sociale au Québec de 1989 à 1996 [36]

Tableau II. Évolution du nombre de pauvres au Québec de 1989 à 1996 [37]

Tableau III. Principales mesures relevant de la sécurité du revenu [38]

Tableau IV. Financement du projet d'un revenu garanti [40]

$\underline{\text { Tableau V. }}$ Hypothèse d'un revenu garanti de 7,500 \$ par adulte, perçue, dans le contexte d'autres données de sécurité du revenu en\% de 1999 [41]

Tableau VI. Montant des impôts reportés au bilan des compagnies, fin de l'année 1999 [42]

Schéma I. Comparaison entre l'impôt négatif et l'allocation universelle du point de vue de leur structure distributive [43] 


\begin{abstract}
Alain MASSOT
sociologue et professeur agrégé,

retraité du Département des fondements et pratiques en éducation,

Université Laval
\end{abstract}

\title{
Une justification du revenu de citoyenneté inconditionnel et universel au dessus du seuil de pauvreté.
}

Communication présentée au 70e Congrès de l'ACFAS, Sociologie et anthropologie. Québec : Université Laval, le 14 mai 2002, 41 pp.

\section{Introduction}

\section{$\underline{\text { Retour à la table des matières }}$}

La thèse centrale de ma communication, bien que vivement discutée et même contestée, repose sur la fin du travail salarié comme centralité dans la révolution de la société informationnelle. Par voie de conséquence, le mode de redistribution d'un revenu décent et relativement stable s'avère de plus en plus aléatoire pour des segments de plus en plus étendus de la population active. Cette instabilité distributive se répercute d'une manière de plus en plus insidieuse sur l'ensemble des citoyens et des citoyennes des pays dits développés. Pour donner une temporalité historique à cette mutation, plusieurs auteurs ont employé diverses formules plus littéraires les unes que les autres telles que : Le troisième âge, la troisième vague, le village global, de la galaxie Gutenberg à la galaxie internet...

Étant donné le niveau de généralité de cette thèse qui se situe au niveau structurel et macro-sociétal, j'avancerai, ici, six propositions générales plus restreintes constitutives de cette mutation historique. 
J'aborderai, ensuite, quelques considérations très générales sur le revenu de citoyenneté et sur certaines de ses retombées anticipées pour finalement mentionner trois modalités d'application du revenu de citoyenneté développées au Québec.

Le revenu de citoyenneté est un revenu distribué égalitairement à toutes les personnes de la communauté politique de référence sur une base individuelle et sans aucune restriction.

J'ouvre, ici, une parenthèse sur la terminologie. Le revenu de citoyenneté n'est pas un revenu minimum garanti. Celui-ci s'adresse seulement aux personnes qui n'ont aucune ressources financières. Le revenu minimum garanti n'est donc pas universel. Il ne s'agit pas non plus un régime d'impôt négatif complémentaire aux revenus de travail dont le salaire serait trop faible ; ce qui correspond approximativement au Régime universel de sécurité du revenu proposé par la Commission royale sur l'union économique canadienne de 1985. L'expression allocation universelle apparaît plus neutre aux yeux de plusieurs, certes, mais le verbe allouer a une connotation de condescendance dans le fait d'octroyer une subvention comme s'il s'agissait d'un avantage, voire, d'un privilège. Un revenu, par contre, est ce qui est dû et perçu comme le fruit d'un travail, d'une activité, d'un capital ou comme le fruit de la richesse collective. Une politique des revenus, par exemple, concerne les actions des pouvoirs publics pour répartir équitablement entre les catégories sociales les revenus provenant de l'activité économique de la nation (cf. définition du Larousse). Parler d'un revenu universel apparaît plus fondamental dans les circonstances car il s'agit d'un nouveau droit-créance, non d'une charité, par le fait même d'appartenir à une communauté politique. Ce revenu se justifie parce que l'on est citoyen d'une nation détenant une certaine richesse. Utiliser l'expression revenu de citoyenneté m'apparaît mieux fondée en regard du droit des citoyens que l'expression allocation citoyenne minimum par exemple, proposée par l'Action démocratique du Québec. Pour un revenu de citoyenneté, universel et inconditionnel, (sans aucune discrimination et sans aucune condition), au-dessus du seuil de pauvreté- et non pas, un strict minimum d'existence pour "assurer le pain et le gîte" selon la version de Charles Sirois, président du conseil d'administration et chef de la direction de Téléglobe, porte-voix du Parti Libéral, qui, lui-même, doit bien gagner un million de fois le pain et le gîte qu'il aimerait garantir à tous, juste pour survivre. On 
aura reconnu la meilleure formule, à mon sens, qui est l'objet du dernier combat politique de Michel Chartrand.

Levons immédiatement une objection : le revenu de citoyenneté n'est recevable que s'il s'arrime à la fiscalité et une politique d'équité fiscale. Pour faire court, ce chèque revient à l'État lorsqu'il est versé à des personnes bénéficiant d'un revenu relativement élevé. Mais alors, pourquoi instaurer un régime universel si son application ne vise qu'une partie de la population. Le principe est universel, ses conséquences sont commandées par la situation réelle des citoyens qui, de plus en plus nombreux et de façon aléatoire, tombent dans la trappe du chômage, de la pauvreté, du bien-être social et de l'exclusion. La recherche de l'équité, face à ce nouvel enjeu, repose sur des fondements historiques, économiques, éthiques et politiques. Nous ferons référence, ici, seulement à des arguments qui relèvent de la nouvelle économie politique du travail .

\section{Une justification d'ordre socio-historique}

\subsection{La réduction du temps de travail}

La réduction du temps de travail est une donnée historique séculaire et irréversible : De 80 heures semaines au début du XIXe siècle, il n'occupe guère que 30 à 35 heures semaines en ce début du XXIe siècle.

\section{$\underline{\text { Retour à la table des matières }}$}

Prenant comme exemple les États-Unis, on peut schématiquement repérer trois étapes de la réduction du temps de travail au cours des deux derniers siècles. La première période s'étend de 1791 aux années 1850, pour la journée de 10 heures ; la deuxième période, du milieu du XIXe siècle à la Deuxième guerre mondiale, pour la journée de huit heures ; la troisième période, de la Deuxième guerre mondiale à aujourd'hui pour les trente-cinq heures ou la semaine de quatre jours. 
Le premier fait recensé dans la chronologie de la réduction du temps de travail aux États-Unis par l'historien américain, Philip Foner, concerne "la première grève pour la journée de 10 heures de travail alors que les travailleurs sont exaspérés par le mode de rémunération en vigueur consistant en un salaire à taux fixe pour les longues journées de travail au cours de l'été et un salaire à la pièce pour les journées de travail écourtées pendant l'hiver.” Cela, en mai 1791. Il faut en comprendre deux choses : s'ils faisaient la grève pour la journée de 10 heures, c'est qu'ils travaillaient plus que dix heures semaine. S'ils contestaient un mode de rémunération différentiel selon les saisons, on comprend qu'ils rejetaient l'instabilité saisonnière de leur salaire. (Cela fait penser aujourd'hui, à la situation précaire des travailleurs saisonniers mal rémunérés par une formule inadaptée et malnommée : l'assurance emploi). La journée de 10 heures sera pratiquement généralisée vers le milieu des années 1850 aux États-Unis.

À partir de cette période, les travailleurs revendiqueront la journée de huit heures. Ces luttes incessantes pour la journée de huit heures dureront un siècle, avec des moments épiques et tragiques comme ceux de 1886 que l'on célèbre le 1er mai (Labor's Emancipation Day) depuis la fondation de la deuxième internationale en 1889. Et des moments moins tragiques, comme par exemple, lorsque le 5 janvier 1914, Henry Ford devient le premier industriel américain à instituer le salaire journalier à cinq dollars et la journée de huit heures. Mentionnons la date buttoir de 1938 alors que le FLSA (Fair Labor Standard $A c t$ ) établit la semaine de cinq jours sur l'horaire de base de 40 heures dans l'industrie à travers les États-Unis.

On ne saurait couvrir cette période sans s'arrêter un instant sur l'épisode unique de la Grande dépression au cours de laquelle la loi des trente heures pour partager l'emploi fut revendiquée, proposée, adoptée en vue d'apporter une solution au chômage massif. Rappelons quelques données brutes : Le chômage qui était inférieur à 1 million en octobre 1929 s'élève à plus de 10 millions en décembre 1931, à 13 millions en juin 1932 et au-delà de 15 millions en mars 1933, atteignant alors un taux record de 24,9\%. Cet épisode de la loi BlackConnery du début des années trente, est rapporté par le groupe de recherche CIRANO dans un ouvrage récent dont l'interprétation s'écarte largement des faits historiques connus. Selon Huberman et Lacroix : “...Les syndicats américains ne s'opposaient pas au partage de l'emploi 
et, durant les années 30, on trouve des exemples de conventions collectives stipulant les conditions et modalités du partage de l'emploi (Jacoby, 1985, 1993). Toutefois, de façon générale, les entreprises utilisèrent le temps réduit de façon discrétionnaire et arbitraire [...] C'est pourquoi, en réponse au président Hoover et aux groupes d'employeurs qui avaient vigoureusement fait la promotion du partage de l'emploi en 1931 et 1932, la plupart des syndicats ont dénoncé ces propositions comme étant essentiellement des moyens de "partager la misère". C'est dans ce contexte que le Black-Connery Bill, qui voulait limiter la semaine de travail à trente heures dans le but de partager les emplois, passa au Sénat mais fut défait en Chambre en 1933.” (Huberman, M. et R., Lacroix, 1996, pp. 26-27).

Cette version des faits diffère largement de celle du sociologue américain Jeremy Rifkin lorsqu'il rapporte ces événements : "Lors des audiences du Congrès sur la loi Black, en janvier et février 1933, William Green, de l'AFL (American Federation of Labor) témoigna de sa certitude que "la réduction de la journée et de la semaine de travail doit être mise en application de façon générale et universelle si nous voulons offrir et créer des chances de travailler à des millions de travailleurs inactifs, et qui ne souhaitent pas le rester"." (Thirty-Hour Week Bill, Hearings on S.5267, 72nd Congress, 2nd Session, pp.1314). “À la grande surprise du pays, le Sénat vota la loi Black le 6 avril 1933, par 55 voix contre 30...Le vote du Sénat enthousiasma le public mais fit frissonner d'horreur Wall Street. "GRANDE VICTOIRE”, clamait en gros titre le journal Labor de Washington. La loi Black fut immédiatement transmise à la chambre des représentants, où William P. Connery Jr, élu du Massachusetts, président de la commission de l'Emploi, lui prédit un vote rapide. La loi fut adoptée en commission, avec recommandation à la Chambre d'en accepter le texte. Son avenir semblait assuré. La plupart des Américains se voyaient déjà les premiers au monde à travailler trente heures par semaine. Mais ce souffle d'espoir allait vite retomber. Le président Roosevelt, soutenu par les principaux responsables industriels du pays, prit immédiatement des mesures pour torpiller le projet...Plus tard, Roosevelt, "émit des regrets pour ne s'être pas rangé derrière la loi Black-Connery sur les trente heures et ne l'avoir pas soutenue devant le Congrès". (Labor, 8 octobre 1935, cité in Roedinger et Foner, p. 252-253 cité par Rifkin, J., 1996, pp. 53 et 54). 
Les auteurs de CIRANO sont en droit d'écrire "que sur ce continent, le partage de l'emploi, fut peu utilisé avant la deuxième guerre mondiale...” (p.18). Les législations américaines des années 30 ont accordé très peu d'attention au partage de l'emploi affirment-ils (p. 27), ce qui semble établit ; mais pas pour les raisons que les auteurs invoquent. Les revendications pour le partage du travail qui précédèrent le New Deal furent détournées par certaines grandes compagnies auxquelles céda le président Roosevelt nouvellement élu. Après le New Deal, le taux de chômage se maintenait encore à 15\% jusqu'en 1940. C'est finalement l'entrée en guerre des États-Unis qui aura raison du chômage puisqu'il tombe à 7,5\% en 1942 et 6,7\% en 1943 (cf. Rifkin, J., 1996, p. 49 et p. 57). La question du chômage technologique ne sera conjoncturellement réglée qu'avec l'entrée des États-Unis dans le deuxième conflit mondial. En 1935, le philosophe Bertrand Russell schématisait le problème : "Il ne faut pas huit heures de travail pour certains et zéro pour d'autres, mais quatre heures de travail pour tous”. Nous en sommes toujours là aujourd'hui.

Après la Deuxième guerre jusqu'aux années quatre-vingt-dix, les revendications pour la réduction du temps de travail aux États-Unis, oscilleront entre la semaine de 36 heures et la semaine de 20 heures de travail, et/ou pour la formule de quatre jours. Dès 1945, les travailleurs des Chemins de fer et de la Marine marchande revendiqueront la semaine de 36 heures. En 1957, la SWU (Steel Workers Union) propose soit la semaine de quatre jours, soit un congé sabbatique de trois à quatre mois tous les cinq ans. En 1961, la Fraternité des électriciens de New-York arrivent à un compromis pour la journée de cinq heures et 25 heures semaines. La convention de l'AFL-CIO de 1963 réaffirme son objectif d'amender le Fair Labor Standard Act pour atteindre la norme de la semaine de 35 heures sans réduction de salaire. Les auditions des Amendements au Fair Labor Standard Act de 1938 visent la réduction obligatoire de la semaine de travail à trente-cinq heures. Cette loi est proposée par le représentant de l'État du Michigan, John Conyers, devant le Comité des Normes du travail et de l'Éducation de la Chambre des représentants en octobre 1979. Mentionnons encore le Full Employment Act proposant la semaine de trente heures présenté au Congrès par le représentant Lucien Black le 14 mars 1994. (Comme on peut le constater, les trente-cinq heures en France ne sont pas une innovation et on se demande pourquoi tant de tohu bohu!). 
Mais revenons aux États-Unis, il est difficile de suivre les auteurs de CIRANO (le Centre interuniversitaire de recherche et d'analyse scientifique des organisations et des comportements stratégiques) lorsqu'ils affirment : Aujourd'hui, “Aux États-Unis, la grande préoccupation, c'est la possibilité de surchauffe du marché du travail et la relance de l'inflation. On est bien loin de se demander si l'on devrait partager l'emploi pour en augmenter le nombre.” (Huberman, M. et R. Lacroix, 1996, p. 17). Étonnant comme constat puisque la réduction du temps de travail au États-Unis, ainsi que dans l'ensemble des pays industrialisés, d'ailleurs, est une lutte séculaire !

\subsection{Le taux de chômage}

Le taux de chômage est une mesure inadéquate du taux d'activité de la population. Il faudrait au minimum le multiplier par deux. De plus, il ne mesure pas la tendance générale à la précarisation des emplois.

\section{$\underline{\text { Retour à la table des matières }}$}

Les statistiques sur le chômage sont loin d'être fiables, titrait le journal Le soleil du 15 février dernier. Cela, on le savait déjà. Mais lorsque c'est le directeur général de l'Institut de la statistique du Québec qui l'affirme devant une commission parlementaire de l'Assemblée nationale, cela fait la manchette : "Moi, je dis aux gens : utilisez toujours avec un grain de sel les taux de chômage régionaux ou pour les régions métropolitaines... On est arrivé à des conclusions, que j'appellerais effrayantes et inquiétantes : si on se fiait à ces données administratives, les taux de chômage doubleraient d'un coup sec” soutient Yves Fortin.

Dans un article intitulé Les chiffres trompeurs du chômage, Francisco Vergara les explicite: "Le taux de chômage est obtenu par un sondage auprès des ménages. On interroge chaque personne pour savoir si elle a travaillé "ne serait-ce qu'une heure" pendant la semaine de référence (en général celle qui précède l'enquête). Si la personne répond par l'affirmative, elle est classée comme ayant un emploi. Sinon, on lui pose une deuxième question : avez-vous "cherché active- 
ment" un emploi lors de la semaine de référence ? Si la personne répond : "oui", une troisième question est alors posée : êtes-vous "immédiatement disponible" pour occuper un emploi ? Seuls ceux qui ont répondu par l'affirmative à ces deux dernières questions sont classés chômeurs.” (Le Monde diplomatique, janvier 1997). C'est la mesure du taux de chômage au sens du BIT (Bureau international du travail). Les experts de l'OCDE ont, eux aussi, noté l'insuffisance de l'indicateur BIT : "Le taux classique de chômage ...ne mesure pas la totalité du sous-emploi." (OCDE, Perspectives de l'emploi juillet 1993, cité par Vergara, F., 1997, p. 17).

Dans son dernier livre, La Fin du travail, Rifkin souligne le même problème en reprenant le constat de l'US Bureau of Labor Statistics, Current Population Survey, 1993 : "En 1993, plus de 8,7 millions de personnes étaient sans emploi ; 6,1 millions travaillaient à temps partiel mais auraient souhaité un temps plein ; plus d'un million étaient si découragées qu'elles avaient cessé toute recherche. Soit un total de 16 millions de travailleurs américains, ou $13 \%$ de la population active sans emploi ou sous-employée." Si l'on cible des sous populations comme les jeunes, il faut alors parler d'un taux de $20 \%$ pour la même année (Rifkin, J., 1996, p. 31, et p. 282).

Le réputé économiste de la Sloane School du MIT, Lester Thurow, ne dit pas autre chose : "Il faut relativiser les statistiques [du chômage aux États-Unis]. Officiellement, le chômage ne touche que 5,5\% de la population, soit 7,5 millions de personnes. Mais il faut en ajouter 6 millions qui se déclarent sans emploi quand on les interroge. Simplement, ils ne sont pas comptabilisés parce qu'aux États-Unis ne sont considérés comme chômeurs que ceux qui ont fait une démarche active pour trouver un emploi dans la semaine précédant le recensement. Si vous êtes resté chez vous parce que vous avez perdu tout espoir de trouver un boulot, vous n'existez pas pour les statistiques. Le chômage, aujourd'hui, est donc plus proche de 10\% que de 5\% estime-t-il.” (Le Nouvel Observateur, 9-15 octobre 1997).

Il y a plusieurs manières de manipuler les statistiques du chômage. La Grande Bretagne aurait réussi à couper en deux le taux de chômage réel sous le règne des conservateurs. Alors qu'il y avait 1,3 millions de chômeurs officiels lors de l'élection de Mme Margaret Thatcher en 1779 , il n'y en aurait que 1,7 million actuellement, soit $6,1 \%$ de la population active. Or, si la méthode de calcul n'avait pas changé, c'est un 
peu plus de trois millions de chômeurs qu'il faudrait recenser. Un rapport de la Middland's Bank, publié récemment, estimait même leur nombre à 4 millions, soit $14 \%$ de la population active (Milne, S., Le Monde diplomatique, mai, 1997, p. 8). Cette même procédure du BIT introduit d'autres biais lors qu'on l'applique à des comparaisons internationales dans des contextes aussi différents que l'Europe et les ÉtatsUnis : "Les experts qui se sont penchés sur les problèmes de comparaisons internationales du chômage ont unanimement reconnu l'insuffisance du taux du BIT” souligne Vergara. Même le ministère du Travail américain l'admet : "Le taux de chômage [au sens BIT] est commode et bien connu; néanmoins, en nous concentrant trop sur cette seule mesure, nous pouvons obtenir une vision déformée de l'économie des autres pays, comparée à celle des États-Unis... D'autres indicateurs sont nécessaires, si l'on veut interpréter de manière intelligente les situations respectives sur les différents marchés du travail.” (International Comparisons of unemployement Indicators, Monthly Labor Review, Washington, mars, 1993, p. 3).

Pourtant, début avril de cette année, la ministre Marois se réjouissait : Le Québec fait chuter le chômage au pays : de 9,3 à 8,9 pour cent ; soit en détail, 7,3\% pour la ville de Québec (un taux réel-officiel de 14,6\%) ; 8,9\% pour Montréal (un taux réel-officiel de 17,8\%); 11,5\% pour Trois-Rivières (un taux réel-officiel de 22\%) ; 12,3\% pour Chicoutimi-Jonquière (un taux réel-officiel de 24,6\%) (Journal de Québec, 6 avril 2002). Ne parlons pas de la Gaspésie où le taux se maintiendrait à plus de 20\% (un taux réel-officiel de plus de 40\%) (Le soleil, 9 avril 2002).

Les statistiques du chômage produisent donc un portrait largement déformé de la situation de l'emploi par la nature même des procédures utilisées. La répétition systématique de données tronquées induisent un effet idéologique très fort sur la perception de la bonne santé de l'état économique. En tout état de cause, il faudrait carrément abandonner la mesure du taux de chômage qui est totalement inadaptée aux nouvelles conditions de l'emploi pour adopter une mesure plus élaborée des formes d'activité et de sous activité qui révélerait la face cachée du chômage à la manière de l'étude sociologique réalisée en France par le Commissariat Général du Plan fin des années 90. Les catégories suivantes furent utilisées : précarité subie ; retrait anticipé d'activité ; ne pouvant chercher un emploi ou découragé d'en cher- 
cher ; travaillant à temps réduit ; demandeur en formation. Ainsi, on obtient un taux d'inactivité et de sous activité mesurant diverses formes de précarité, ce qui multiplie par deux le taux officiel du chômage pour un total de 7 millions de personnes en France. Les auteurs du rapport -Robert Castel, Jean Fitoussi, Jacques Freysinnet et Henri Guaino- concluent: "C'est toute la structure du travail qui est en train de se modifier vers plus d'insécurité pour toutes les catégories...Les quelques 3 millions de chômeurs au sens du bureau international du travail ne forment que le noyau dur d'un vaste phénomène de décomposition de la relation au travail.” De plus, cette étude avait l'immense mérite de mesurer le taux d'activité d'une manière longitudinale. Les auteurs du rapport proposent une nouvelle mesure du chômage de longue durée : "Il s'agirait de prendre la moyenne des périodes cumulées de chômage sur trois ans et non plus seulement les douze mois continus d'inscription à l'ANPE dans la dernière année. Résultat ? La part des chômeurs de longue durée passerait en 1996 de 35 à 64\% !" (L'Express, 16-22 octobre 1997). Les personnes sont amenées à côtoyer le chômage tout au long de la vie. Le diplômes protègent, mais de moins en moins. Or, la part des surdiplômés ne cesse d'augmenter ! Les auteurs ajoutent : "Ce n'est pas le chômage lui-même qui fait problème mais la peur de ne pas s'en sortir indemne, la peur des ruptures, des régressions, de la dégradation du capital humain.”

Quant à la peur du chômage aux États-Unis, "Deux travailleurs américains sur cinq craignent d'être licenciés, ou d'être forcés d'accepter des réductions d'horaires ou de salaire, dans les deux années suivantes. Soixante-dix-sept pour cent des personnes interrogées disent connaître parmi leurs relations directes une personne ayant perdu son emploi ces dernières années, et 67\% affirment que le chômage a des répercutions sensibles dans leur entourage." (From Coast to Coast, from Afluent to poor, Pool Shows Anxiety Over Jobs”, New York Times, 11 mars, 1994, cité par Rifkin, J., 1996, p. 33).

Rien de cela n'autorise l'optimisme béat que pourrait susciter la dite situation de plein emploi aux États-Unis et encore moins la construction d'une analyse comparative concluant à l'éternel recommencement du "miracle américain." Il est vrai que le taux officiel du chômage aux États-Unis qui se tenait à $3 \%$ dans les années cinquante, s'établissait à 6,7\% au début de la décennie quatre-vingt-dix. Il prend figure maintenant de "taux naturel" du chômage tel que reconnu par 
les économistes. Le taux naturel est défini comme étant le taux audelà duquel, l'économie aurait une tendance inflationniste. Au Canada, des experts viennent de décréter que le "taux naturel" devrait s'établir à $7 \%$.

\subsection{La logique de l'investissement}

\section{Le niveau de l'emploi est une fonction décroissante de l'investissement dans la mutation de la société informa- tionnelle.}

$\underline{\text { Retour à la table des matières }}$

Depuis longtemps, la science économique conventionnelle répète la même litanie : les technologies stimulent la productivité, abaissent les coûts de production, augmentent l'offre des marchandises. Cela élève le pouvoir d'achat, étend les marchés et génère des emplois supplémentaires...

Une grande partie de la théorie économique classique tourne autour de cette argumentation, que ce soit la loi des débouchés (l'idée qu'un nouveau produit crée un débouché pour d'autres produits) ; que ce soit la loi du cycle productiviste (toute perte d'emplois résultant de l'automation sera compensée par une nouvelle demande de biens de consommation) ; que ce soit la loi du déversement selon laquelle une diminution de l'employabilité dans un secteur hautement technicisé engendre une nouvelle demande de main-d'oeuvre dans de nouveaux secteurs d'activité. Et enfin, un dernier argument de ce discours officiel : même si l'automation supprime massivement de l'emploi, cela ne peut qu'exercer une pression à la baisse des salaires, ce qui conduira à un réajustement du coût de la main-d'oeuvre, à de nouveaux investissements technologiques et, au bout du compte, à un équilibre de l'offre et de la demande.

Cette argumentation demeure en partie fondée. L'histoire économique moderne s'explique pour une part par un tel modèle. Il serait bien naïf de l'invalider totalement ; ne pas investir, c'est atterrir sur le plancher zéro de l'emploi. Mais dans la conjoncture actuelle, nous sommes sollicités constamment par deux propositions contradictoires, 
l'une dominante que l'on vient d'évoquer: l'investissement crée de l'emploi ; l'autre marginalisée : l'investissement (dans les nouvelles technologies) supprime de l'emploi. La confusion qui entoure la relation entre les investissements et la création d'emplois se dissiperait si l'on apportait une réponse satisfaisante à cette contradiction apparente.

Généralisons :

1) L'investissement dans les nouvelles technologies supprime plus d'emplois qu'il n'en crée.

2) L'automation permet d'économiser du capital.

3) L'automation augmente la productivité.

4) Il n'est plus possible d'attendre de la relance de l'investissement une solution au problème de l'emploi.

En d'autres termes, le niveau de l'emploi est une fonction décroissante de l'investissement dans le contexte de la mutation technologique. Charles Levinson le disait il y a déjà 30 ans : l'investissement n'est plus créateur, mais destructeur d'emplois.

Une constatation s'impose : il n'est plus possible d'attendre de la relance de l'investissement une solution au problème de l'emploi. C'est la fin du travail à plein temps pour tout le monde. Une conclusion s'en dégage : toute politique de travail pour tous passe par une réduction du temps de travail, et, plus globalement, par une nouvelle politique de répartition du temps de travail socialement nécessaire. 


\subsection{Le mode de production post-fordiste}

La logique de l'investissement du mode de production post-fordiste engendre une externalisation de la maind'oeuvre sous la forme d'un post-salariat précarisé de plus en plus étendu à la périphérie d'un noyau central de travailleurs permanents, polyvalents et à temps plein de plus en plus restreint.

Je me référerai de nouveau aux États-Unis en ce qui concerne la bipolarité indissociable création/destruction d'emplois. Les données et les projections concernant la destruction d'emplois sont plus dévastatrices que celles relatives au chômage et d'un tout autre ordre de grandeur puisqu'il s'agit de La Fin du travail, titre d'un livre de Michel Drancourt publié en 1984 et repris par Rifkin en 1996.

Entre 1956 et 1962, plus de 1,5 millions d'ouvriers perdirent leur travail aux États-Unis, alors que ces dix dernières années plus de 3 millions d'emplois de bureau ont été éliminés, et qu'actuellement, deux millions d'Américains perdent chaque année leur travail (Rifkin, J., 1996, p. 103, p. 29 et p. 63). Le très sérieux Wall Street Journal, affirme que le reengineering pourrait éliminer 1 à 2.5 millions emplois par an "à échéance prévisible” dans l'économie américaine : "Tandis que le reengineering fait ses premiers pas, certaines études prédisent une perte totale de 25 millions d'emplois dans le secteur privé, dont l'effectif actuel approche les 90 millions”. Selon Michael Hammer, ancien professeur au MIT, les investissements massifs dans le reengineering "entraîne généralement la suppression de plus de $40 \%$ des emplois d'une société ordinaire, et jusqu'à 75\% dans certains cas particuliers...la proportion de cadres moyens susceptibles de voir leurs postes disparaître pouvant atteindre 80\%." Cette révolution technétronique signifie que "pour les seuls États-Unis... dans les années à venir, plus de 90 millions d'emplois, sur une population active totale de 124 millions de personnes, pourraient être remplacées par des machines.” (Rifkin, J., 1996, p. 26 et p. 24). 
Quant à la création d'emplois, le portrait de la situation ne laisse guère de place à plus d'optimisme : "Entre 1969 et 1994, le nombre des travailleurs à temps partiel subi est passé, aux États-Unis, de 6\% à 12,9\% de la population active. La part des salariés mal payés (moins de 15000 dollars par an) a triplé, passant de 8,4\% à 32,2\%. Celle des pauvres qui travaillent (les Working poor) et des chômeurs a crû de $22,9 \%$ à 38, 5\% du total. Si plus du tiers des actifs américains vivent ainsi dans la pauvreté, cela tient, pour la majorité d'entre eux, à la faiblesse de leurs salaires, et non pas à leur dépendance à l'égard de l'aide sociale.” (Petras, J., et T. Cavaluzzi, 1996, p. 11). Ce que confirme Rifkin : "En 1994, les deux-tiers des emplois créés se situaient au bas de l'échelle des salaires.” Selon une étude du ministère du Travail, moins de $20 \%$ des personnes recyclées dans le cadre des programmes fédéraux de lutte contre le chômage réussissent à trouver un nouvel emploi leur apportant au moins 80\% de leur ancien salaire. (cf. Rifkin, J., 1996, p. 22 et p. 63). Cette situation n'échappe plus aux plus ardents défenseurs du libéralisme. Selon le sénateur M. Bill Bradley, la fureur compétitive, la précarisation de l'emploi et la baise des salaires font que "les classes moyennes américaines vivent de plus en plus mal et doivent travailler de plus en plus pour maintenir leur niveau de vie.” (Ramonet, I., 1996, p. 1). Le président William Clinton, résumait la situation sociale : "Trop nombreux sont nos citoyens qui travaillent toujours plus pour gagner toujours moins et pendant que nos entreprises se restructurent et deviennent de plus en plus concurrentielles, ces Américains ne savent pas s'ils auront encore un emploi l'année prochaine, ou même le mois qui vient. (...) Si nous ne réagissons pas, notre économie continuera à faire ce qu'elle fait depuis 1978 : elle procurera des revenus croissants à ceux qui sont au sommet, offrira très peu à ceux qui sont au milieu et laissera ceux qui sont en bas continuer à chuter même quand ils travaillent plus dur.” (Cité par Halami, S., 1996, p. 3).

Le soi-disant succès des États-Unis en matière de plein emploi ne résiste pas à l'analyse critique des taux de chômage. Le discours lénifiant sur la logique économiste conventionnelle de l'investissement conduisant au plein emploi est invalidée dans le contexte précis de la mutation technologique. Il en est de même en ce qui concerne la création d'emplois au États-Unis alors qu'il faut traiter de la destruction 
massive d'emplois, de précarité et d'exclusion largement documentée dans de nombreuses études disponibles.

D'autres projections confirment l'ampleur de la mutation. "Dans les cinq cents plus grandes sociétés américaines, la proportion des emplois permanents et à plein temps ne représente plus que $10 \%$ du total. La deuxième banque des États-Unis par ordre d'importance (la Bank America) se réorganise de manière à ne conserver que $19 \%$ de personnel stable et à plein temps, les $81 \%$ restants, de tous les niveaux de qualification, n'étant employés qu'à titre précaire, de façon intermittente et à temps partiel, moins de vingt heures par semaine dans 60\% des cas.” (Aznar, G., 1993, p. 12). Même ordre de prédiction pour Robert Reich : “... Sur le marché du travail, le rapport sera bientôt de quatre emplois secondaires (peu qualifiés et peu rémunérés) pour un emploi primaire (fortement qualifié et fortement rémunéré).” (Reich, R., B., 1992).

Au même moment, la productivité des entreprises manufacturières a augmenté de 35\% de 1979 à 1992 aux États-Unis, tandis que le nombre des employés a diminué de $15 \%$. Rappelons que $90 \%$ des emplois créés aux États-Unis au début de 1993 étaient des emplois temporaires ou à temps partiel. Les cinq cents plus grandes firmes américaines n'emploient que $10 \%$ des salariés permanents et à plein temps (Aznar, G., 1993, p. 13). (Là encore, les statistiques officielles sur la perte d'emplois et la création d'emplois exercent un fort effet idéologique si l'on ne prend pas en compte le statut des emplois.)

C'est la raison principale pour laquelle le plein emploi devient un objectif qui ne cesse de s'éloigner. Dans un certain sens, il s'agit là d'une bonne nouvelle car, après tout, pourquoi se plaindre du fait que les machines peuvent travailler à notre place !

Ce qui se profile derrière ces données convergentes, c'est le modèle post-fordiste qui engendre une externalisation de la main-d'oeuvre et qui transforme en profondeur les rapports de classes au sein même des entreprises. Schématiquement, on voit se constituer au coeur des entreprises un noyau central d'employés stables, polyvalents participant pleinement à l'entreprise. Ce noyau est parfaitement illustré par les investissments réalisés par une compagnie comme l'Alcan qui fait des investissements de 2 milliards de dollars générant la création de deux cents emplois (ce qui fait un nouvel emploi créé pour chaque dix mil- 
lions d'investissement). Autour de ce noyau, se développe une main d'oeuvre périphérique peu qualifiée renouvelable au gré de la demande et alimentée par une armée de réserve d'intérimaires recrutés selon les fluctuations du marché. À la périphérie de ce cercle, on trouve une main d'oeuvre externe qualifiée et non-qualifiée ; ce sont les travailleurs autonomes et flexibles, à leur compte et à leurs risques et périls dont le mode d'embauche le plus perfectionné correspond à la formule du contrat zéro heures (zero hour contract) : "L'employé n'est pas employé mais tenu d'être en permanence à la disposition de l'entreprise, en attendant qu'on ait besoin de lui pour quelques jours, ou quelques heures au tarif horaire convenu." (André Gorz, 1997, p. 152). Un exemple typique est le mode d'embauche pour l'Exposition Universelle 2002 de Neufchâtel. L'employeur a à offrir 20 millions d'heures pour quelques trois mille travailleurs rémunérés à 18 francs pour les employés non-qualifiés et 22.25 francs pour les employés qualifiés sur une durée de six mois. Le critère d'embauche incontournable est la flexibilité. Enfin, à l'extrême périphérie, se développe la masse des exclus, des pauvres, des itinérants qu'alimentent les cohortes de décrocheurs.

Les statistiques sur création d'emploi sont indissociables de celles sur la destruction d'emploi et elles doivent rendre compte du statut des emplois. Dans le cas contraire, elles ne signifient pas grand chose.

\subsection{Le dépassement de la dualisation sociétale}

La fin du travail mène droit à la dualisation de la société. La distribution de la richesse collective ne permet pas un niveau de vie décent à une part toujours plus grande de la population. La réduction drastique du temps de travail couplée au partage du travail peuvent être envisagés comme un dépassement de la dualisation sociétale.

\section{$\underline{\text { Retour à la table des matières }}$}

De la même façon irréversible que la machine a relégué la force animale aux oubliettes, l'automation va dissoudre le travail humain dans ses configurations actuelles. Évitons encore un contresens possi- 
ble sur cette thèse de la fin du travail. Les gisements de travail sont immenses ne serait-ce que pour satisfaire au minimum d'existence décent au-dessus du seuil de pauvreté. Le problème majeur est que cette mutation semble bien mal négociée si tant est que l'on puisse négocier une telle mutation historique.

La réduction du temps de travail crée de l'emploi. À preuve, le temps de travail annuel a été réduit de moitié depuis la révolution industrielle, de quatre-vingts heures à soixante heures semaine au cours du XIXe siècle et jusqu'à quarante heures à partir de la deuxième moitié du XXe siècle. Il faut prendre en compte également le fait que les jeunes de 8 à 25 ans et les aînés sont largement sortis du marché du travail. Que serait alors le taux d'inactivité si les horaires de travail n'avaient pas baissés drastiquement au cours du développement de la technologie et si des strates d'âge importantes faisaient encore partie de la population active?

Les mesures défensives de réduction du temps de travail créent de l'emploi. L'exemple de Volkswagen est devenu célèbre : les aménagements du temps de travail ont permis d'éviter le congédiement de 30000 travailleurs. La semaine de quatre jours a été proposée en France et selon le plan Larrouturou. Cette semaine de trente-trois heures entraînerait une réduction de $5 \%$ des salaires, mais créerait deux millions d'emplois (soit une hausse de l'emploi total de 10\%). L'agrégation de mesures défensives de réduction du temps de travail conduit à un bilan positif en termes de création nette d'emplois. "À ce stade, souligne encore Rifkin, un nombre croissant d'observateurs suggèrent qu'une nouvelle réduction de la semaine de travail, à trente ou même à vingt heures, est inéluctable pour adapter les besoins en travail aux nouvelles performances du capital.” (1996, p. 297).

Par contre, seule une politique de réduction du temps de travail couplée à une politique du partage du temps de travail peut avoir un effet massif sur le chômage. Lynn Williams, ancien président du Syndicat de la métallurgie (USWA), estime que "nous devons commencer à penser à la réduction du temps de travail[...] comme moyen de partager les gains de productivité.” Le secrétaire-trésorier de l'ALF-CIO ne pense pas autrement: "Il est indéniable que l'avenir à long terme du travail dépend de la réduction du temps de travail.” (Rifkin, J, 1996, p. 299, 297 et p. 306). La Commission européenne et le 
Parlement européen s'engagent dans cette voie. Alors qu'il y a un large mouvement de l'opinion publique en faveur de telles mesures, la plupart des chefs d'entreprises américaines y sont encore réfractaires au nom de la sacro-sainte compétivité dans le contexte de la mondialisation.

Aucune autre politique que la réduction substantielle de la semaine de travail et les stratégies offensives d'aménagement du travail ne permettront d'obtenir la création d'emplois à la mesure du niveau de chômage actuel : un problème de 1, 5 millions de chômeurs au Canada et de 425000 au Québec ; un problème de 9,3 millions aux ÉtatsUnis ; un problème de 3,3 millions de chômeurs déclarés en France, (plus 1,5 million de stagiaires et RMIstes, plus les chômeurs découragés de rechercher un emplois : soit un problème de 7 millions pour la France ; un problème de 18 millions de chômeurs comme tels(12\%) dans l'Union européenne ; de trente-cinq millions dans les pays industrialisés ; et un problème de plus de 800 millions d'êtres humains sans emplois sous employés dans le monde.

L'évolution du chômage technologique est irréversible. Elle est inscrite dans la troisième révolution technétronique. L'idéologie dominante occulte cette tendance historique structurelle dans une vision à court terme. Pourtant le père de la cybernétique en avait mesuré l'exacte portée : "N'oublions pas, disait Norbert Wiener, que la machine automatique[...] est l'équivalent exact du travail servile. Toute forme de travail qui veut rivaliser avec l'esclavage doit accepter les conséquences économiques de l'esclavage.” (Rifkin, J., 1996, p. 118). Jacques Robin en explicite les conséquences : "Le saut qualitatif que représentent ces technologies "informationnelles" retentit de manière révolutionnaire sur la marche des sociétés développées... C'est cette mutation radicale qui reste ignorée ou occultée de manière scandaleuse par les responsables... Non seulement le rapport de l'homme à la machine est bouleversé -le travail cesse en grande partie d'être matériel- mais encore les mécanismes économiques et sociaux traditionnels se dérèglent progressivement : la productivité marginale, fondement du calcul économique classique, devient peu significative ; des indicateurs, tels ceux du produit national brut (PNB), de l'indice de productivité, des niveaux d'inflation, ne cadrent plus avec la réalité économique...(1993, p.12). Ainsi, selon Hautecoeur, “... la crise du travail a ébranlé un monde de représentation et un ensemble de pratiques cultu- 
relles qu'on croyait immuables : le droit au travail, la promotion socioprofessionnelle, le rapport éducation-position sociale, certains privilèges d'âge (anciens et jeunes), les critères de compétences reconnues au travail, des traditions de métiers, la familiarité avec le monde des outils, des techniques de production et de communication, etc. À l'école, si "le niveau monte", les taux d'abandons montent aussi ainsi que "le niveau de découragement". L'écart s'agrandit entre les qualifiés et les disqualifiés pour qui la formation n'est plus un espoir de mobilité, mais au contraire, une confirmation de déclassement.” (Hautecoeur, J.-P., UNESCO/MEQ, 1996, p. 399). Le problème s'énonce ainsi : "Si l'on admet qu'il existe un déséquilibre inévitable entre l'offre d'emploi et la demande d'emploi que ne peuvent résoudre ni la croissance ni la productivité, il n'existe que deux voies pour le résoudre : ou bien on s'adapte à une forme de segmentation sociale (ceux qui travaillent, ceux qui ne travaillent pas) en rendant cette situation viable ; ou bien on cherche à redistribuer le travail sur l'ensemble des citoyens.” (Aznar, G., 1993, p. 73).

Face à la dualisation de la société comme résultat inexorable du progrès, nous sommes donc en présence des choix suivants :

1) Ou bien l'on s'en accommode sous réserve de vivre avec le syndrome de Los Angeles et les ghettos urbains. Cette solution déchargerait les privilégiés du poids moral du monopole du travail tout en achetant une paix sociale relative. Parfaitement réaliste, cette solution n'en est pas moins inéquitable et instable.

2) L'autre branche de l'alternative porte sur la redistribution du travail, laquelle s'appuie simultanément sur deux stratégies: l'aménagement et la réduction du temps de travail dans l'optique du partage du travail, et la réinvention d'activités dans un tiers secteur hors de la bipolarisation économie marchande/Étatprovidence.

La recherche des accommodements à la société duale renforce la précarisation et l'exclusion et, par là, contient en elle-même, une structure profondément inéquitable avec des effets catastrophiques à moyen et long termes. Bien plus que de chercher des accommode- 
ments à la société duale, la redistribution du travail suppose un nouvel aménagement général du temps de travail dans le cadre plus global de la problématique du temps choisi, ouvert sur un tiers-secteur, l'économie solidaire notamment, conduisant à plus de liberté, d'équité et de solidarité et d'autonomie.

\subsection{La révolution du temps choisi}

Une discontinuité croissante s'instaure entre le temps de formation et le temps de travail si bien que la séquence formation/travail/retraite-ne concerne qu'une part de plus en plus congrue de la population. La révolution du temps choisi se présente comme le dépassement de la société duale.

\section{$\underline{\text { Retour à la table des matières }}$}

La société de travail est morte dit sans ambages André Gorz. Or, "Jamais, l'idéologie du travail- valeur n'a été affichée, proclamée, ressassée aussi effrontément et jamais la domination du capital, de l'entreprise sur les conditions et le prix du travail n'a été aussi indiscutée. Jamais la fonction "irremplaçable", "indispensable" du travail en tant que source de "lien social”, de "cohésion sociale”, d'“intégration”, de “socialisation”, d'“identité personnelle”, de sens n'a été invoquée aussi obsessionnelle ment que depuis qu'il ne peut plus remplir aucune de ses fonctions... L'emploi cesse d'intégrer dans un collectif, il cesse de structurer le temps quotidien, hebdomadaire, annulent les âges de la vie, il cesse d'être le socle sur lequel chacun peut construire son projet de vie.” (André Gorz, 1997, p. 98). Il en résulte une dualisation sociale incommensurable entre l'accumulation de la richesse et l'extension de la pauvreté. Au même moment, jamais a-t-on vu les études d'insertion se multiplier en aussi grand nombre ! Comme si l'on pouvait insérer le manche d'un marteau dans le chas d'une aiguille !

L'externalisation de la main-d'oeuvre peut prendre d'autres formes que la flexibilité, la précarisation, l'exclusion engendrée par les diktats de l'entreprise. "Toutes les formes de discontinuité subie de l'emploi, de la flexibilité des effectifs et des horaires sont à transformer en pos- 
sibilité de choisir et d'autogérer la discontinuité et la flexibilité.” (André Gorz, 1997, p. 156). Une illustration en est donnée par le système danois orienté sur les principes d'une autre économie :

- La reconnaissance que le droit au travail et le droit au nontravail sont d'égale importance et indissociablement liés.

- La reconnaissance que le droit de travailler de manière discontinue doit s'accompagner du droit à un revenu continu.

Globalement, le travail qui est massivement aboli, dans la production, dans la société globale, dans le monde, “conserve dans la conscience de chacun sa centralité”, alors que le précaire est devenu la figure centrale. Citons encore André Gorz: "Nous nous savons, sentons, appréhendons chacun comme chômeurs en puissance, sousemployé en puissance, précaire, temporaire, "temps partiel" en puissance. Mais ce que chacun de nous sait ne devient pas encore - et est empêché de devenir - conscience commune à tous de notre commune condition.” (André Gorz, 1997, p. 89). “Toutes les puissances établies s'opposent à cette reconnaissance et à ce qu'elle entraîne ... Qu'alors même que le post-fordisme, la mise en réseau de fabriques fractales et l'économie de l'immatériel reposent sur une production de richesses de plus en plus déconnectée du travail et une accumulation de profits de plus en plus déconnectée de toute production, le droit de chacun à un revenu suffisant, le droit à la citoyenneté plénière, le droit d'avoir des droits restent, eux, connectés à l'exercice d'un travail mesurable, classifiable, vendable... Voici donc le coeur du problème et le coeur du conflit : il s'agit de déconnecter du travail le droit d'avoir des droits et notamment le droit à ce qui est produit et productible sans travail, ou avec de moins en moins de travail. Il s'agit de prendre acte de ce que ni le droit à un revenu, ni à la citoyenneté plénière, ni l'épanouissement et l'identité de chacun ne peuvent plus être centrés sur et dépendre de l'occupation d'un emploi. Et changer la société en conséquence.” (André Gorz, 1997, p. 90). Mais comme le dit lucidement, Edgar Morin, il ne faut pas attendre de la science économique des "miracles" tant elle est assujettie aux a priori clandestins de la pensée : "La science économique, qui est la science sociale mathématiquement la plus avancée, est la science socialement et humainement la plus arriérée, 
car elle s'est abstraite des conditions sociales, historique, politique, psychologiques, écologique inséparables des activités économiques... Du coup, l'erreur économique devient une conséquence première de la science économique.” (Edgar Morin, 2000, p. 43).

Les diagnostiques sont posés, les analyses accessibles, les conclusions tirées et les avertissements ne manquent pas. "Le chômage de masse et une récession économique aussi grave que celle des années 30 propulsent les sociétés d'Occident vers des univers cauchemardesques et menacent le minimum de cohésion qu'avait su bâtir l'Étatprovidence dans les années d'après-guerre” (Cassen, B. , 1993, p.1). Madame Rosabeth Moss Kanter, ancienne directrice de la Harvard Bussiness Review écrit : "Il faut créer la confiance chez les salariés, et les organiser la coopération entre les entreprises afin que les collectivités locales, les villes et les régions bénéficient de la mondialisation. Sinon nous assisterons à la résurgence de mouvements sociaux comme nous n'en avons jamais vu depuis la seconde guerre.” (cité par Ramonet, I., 1996, p. 1).

Mais cette révolution technologique ne se fera pas sans concession et sans l'abandon d'un privilège : "Une démocratie ne peut abandonner aux entreprises le monopole des décisions qui, par l'informatisation de la production, jettent sur le pavé des millions de chômeurs dont la charge incombe ensuite à la collectivité nationale. Repenser le travail humain et la vie en société, réviser des "lois" économiques largement invalidées par des mutations sans précédent, négocier de nouvelles normes pour les échanges internationaux : c'est inventer un autre mode de développement, faute de quoi "la misère du monde" engendrera de brutales convulsions qui n'épargneront aucun pays de la planète.” (Julien, C., 1993, p.9).

"Des solutions existent : la réduction massive de la durée du travail pour enrayer la machine à exclure...le renforcement des services publics, le développement d'un tiers secteur d'utilité sociale pour raviver les racines de l'intégration, le développement d'une démocratie de face-à-face, fondement d'un partenariat... Nous ne manquons pas de solutions. Nous manquons de volonté politique”. (Lipietz, A. 1996, p.10). L'utopie sera blanche ou noire prévient aussi Jeremy Rifkin. C'est le point de chute d'une justification socioéconomique de revenu de citoyenneté. 


\section{Un revenu de citoyenneté pour tous}

Retour à la table des matières

La science économique s'est abstraite des conditions sociopolitiques. Pas entièrement, puisque plusieurs partis politiques actuels au Québec et au Canada se mobilisent d'une façon plus ou moins crédible autour de ce principe. Des événements récents témoignent de cette nouvelle donne : Le Rassemblement pour l'alternative politique (RAP) vient d'adopter cette politique dans un atelier de son congrès fondateur tenu à l'Université Laval en novembre 2000. Le Parti libéral du Canada semble avoir lancé un ballon politique sur cette question au lendemain de sa réélection en novembre 2000. Le Nouveau parti démocratique en fait la promotion. Charles Sirois y adhère et le parlement virtuel des aînés du Québec en ont fait leur premier projet de loi. Le principe est enfin à l'agenda politique au Québec.

Le revenu de citoyenneté fait l'objet d'un vaste débat sous d'autres horizons et se trouve déjà intégré explicitement dans les programmes de plusieurs partis politiques en Europe. Il est même question d'un revenu mondial de citoyenneté "étant donné que l'on aurait droit à ce revenu d'existence parce qu'on existe, et non pour exister. Son instauration repose sur l'idée que la capacité productive d'une société est le résultat de tout le savoir scientifique et technique accumulé par les générations passées. Aussi les fruits de ce patrimoine commun doivent-ils profiter à l'ensemble des individus, sous la forme d'un revenu de base inconditionnel.” Ce ne sont pas là les paroles d'un lacédémonien, mais celles d'Ignacio Ramonet, directeur du Monde diplomatique, (janvier, 2000). Les professeurs Jan Tinbergen et James E. Meade, tous les deux prix Nobel d'économie, ont déjà proposé un revenu universel mondial.

Trois objections majeures sont avancées en regard du revenu de citoyenneté. L'une strictement budgétaire et fiscale concerne son impact réel sur le revenu des personnes ; la deuxième concerne le financement global du revenu de citoyenneté ; la troisième objection est strictement d'ordre moral et idéologique. 
Sur le plan personnel, Van Parijs a démontré de façon probante qu'un revenu de citoyenneté, peu importe le montant par ailleurs, ne change rien sur le revenu net disponible des contribuables indépendamment du fait que les montants de transferts soient plus importants.

Pour ce qui est du financement global du revenu de citoyenneté, il faut comprendre que cette politique doit être estimée sur la base du coût net, c'est-à-dire, le coût brut moins les programmes et les régimes existants d'assurance et d'assistance sociale. Remarquons, au passage, que les principaux programmes de sécurité du revenu (Assuranceemploi, Régime des rentes, Régime de compensation des accidents du travail) dépendent de la participation des bénéficiaires au marché du travail. Or, c'est précisément le marché du travail qui vacille. D'autre part, le financement du revenu de citoyenneté doit s'arrimer à la capacité financière globale de l'État en tenant compte non seulement du Budget régulier de l'État, mais aussi de son Budget des dépenses fiscales. Le financement du revenu de citoyenneté est impensable sans une politique fiscale adaptée.

La troisième objection est la plus fondamentale car elle appelle à une révolution des mentalités. André Gorz avait parfaitement anticipé cette difficulté, il y a vingt ans déjà : La résistance à la réduction du temps de travail ne serait donc pas d'ordre économique, mais essentiellement idéologique. "Le chômage est facteur d'ordre" dit Gorz. Dans un article qui commence à dater, sans vieillir, Michel Bosquet visait très justement le point sensible : "Si tout le monde prenait conscience qu'il n'y a plus virtuellement de problèmes de production mais seulement un problème de distribution - c'est-à-dire de partage équitable dans toute la population du travail socialement nécessaire - le système social aurait de rudes difficultés à se maintenir. Que deviendraient la discipline au travail, l'éthique du rendement, l'idéologie de la compétition si chacun savait qu'il est techniquement possible de vivre de mieux en mieux en travaillant de moins en moins, et que le droit à un "plein revenu” n'a plus besoin d'être réservé à ceux qui fournissent un "plein travail ?” (1978).

"La "véritable économie" aboutit à l'élimination du travail comme forme dominante de l'activité, son remplacement par l'activité personnelle qu'il s'agit désormais de vouloir politiquement et de rendre tangible par des changements dès à présent réalisables.” (André Gorz, 1997, p. 151). 
Face à cette nouvelle conjecture structurale contradictoire, nous pouvons anticiper les effets à la fois immédiats et de plus longue durée du principe du revenu de citoyenneté universel et inconditionnel :

1. La pauvreté zéro

2. L'éradication de la misère sociale pour deux millions de Québécois

3. Le respect de la personne

4. L'aide aux parents

5. L'agrandissement du champ de liberté pour les femmes

6. Le libre-choix pour la première génération de jeunes de leur formation, leur métier, leur travail

7. Un nouvel argument de négociation pour les travailleurs et les travailleuses

8. Un frein à l'exode des régions

9. Une impulsion immédiate et continue sur l'économie de marché conventionnelle

Il existe trois scénarios du revenu de citoyenneté au Québec : un programme fort, un programme minimaliste et un programme intermédiaire.

Un programme minimaliste d'allocation universelle inconditionnel est développé par François Blais dans la ligne de pensée de Van Parijs. Il propose une allocation non imposable d'environ 300 dollars par mois et par personne ; celle-ci serait financée, entre autres, par une diminution équivalente de l'aide sociale et l'abolition de l'exemption personnelle de base du Régime de l'impôt sur le revenu. L'allocation pourrait être cumulable avec les revenus de travail. (Cf. François Aubry, p. 34, 1999).

Le modèle intermédiaire propose comme hypothèse un Revenu Garanti annuel de \$7,500 pour chaque adulte. Les enfants seraient exclus de ce modèle dans la mesure où la plupart des programmes les concernant resteraient inchangés. La plausibilité de ce financement 
repose sur la capacité financière globale du gouvernement en tenant compte du budget régulier du Fédéral, mais aussi de son budget des dépenses fiscales. Selon l'auteur de ce modèle, Jean-Baptiste Bergevin, ce projet peut se financer à même les budgets existants, en respectant la structure d'imposition du système fiscal. Il favoriserait la flexibilité de la main-d'oeuvre tout en conservant le facteur de l'incitation au travail. (cf. 2001, p. 3).

Le programme fort est proposé par Michel Bernard et Michel Chartrand dans un manifeste Pour un revenu de citoyenneté au dessus du seuil de pauvreté. On comprend que le seuil de pauvreté serait le revenu minimum. Il faut comprendre aussi que ce programme reposerait en bonne partie sur le redéploiement des budgets des programmes conditionnels actuels. Si bien qu'il faut s'arrêter au coût net plutôt qu'au coût brut du programme. Il faut également en évaluer les effets positifs sur l'économie et les effets positifs en termes d'épargne collective liée à l'éradication de la pauvreté estimée à plusieurs centaines de millions de dollars au Canada. Il faut également évaluer les coûts engendrés par l'exode des régions pour ne mentionner que ceux-là. Il faut également tenir compte des exemptions fiscales et des reports d'impôts tels que documentés par Léo-Paul Lauzon (2002).

\section{Conclusion}

\section{$\underline{\text { Retour à la table des matières }}$}

Il existe une analogie profonde entre la revendication pour le revenu de citoyenneté et les batailles pour l'instruction publique et l'assurance-santé universelles qui ont été pensées afin de soustraire la personne d'une relation de dépendance. Le revenu de citoyenneté est un principe universel et révolutionnaire en ce qu'il constitue une nouvelle condition sine qua non de l'autonomie des citoyens.

Selon Jacques Ellul, la logique technicienne a un développement causal non-finalisé selon une rationalité instrumentale intrinsèque orientée vers une société de consommation illimitée qui dévore le travail. L'inversion de cette logique technicienne ne peut être constituée que par les cinq éléments suivants : 
- Une reconversion totale de la puissance productive du monde occidental orienté vers le développement autonome du tiersmonde ;

- le choix délibéré de la non-puissance ;

- Le parti-pris de la diversification des cultures, des groupes, des partis, des syndicats, des médias ; une condition de l'autogestion ;

- Une réduction drastique du temps de travail ; (Adret a raison lorsqu'il parle de deux heures de travail par jour, c'est la visée immédiate et immédiatement réalisable malgré les cris des réactionnaires). Cela implique une réorganisation du travail ouverte sur le temps choisi.

Cette inversion de la logique technicienne implique une mutation dans la répartition de la richesse collective. "Une révolution était nécessaire pour supprimer l'esclavage ou pour supprimer le prolétariat. Une révolution est actuellement nécessaire en face de l'État et de la Technique autonome. Nous sommes maintenant face à cette révolution là, et pas d'une autre.” (cf. Ellul, Jacques, 1977). 
Références

$\underline{\text { Retour à la table des matières }}$

AUBRY, François, L'allocation universelle. Fondements et enjeux, Montréal, CSN, 1999.

AZNARD, Guy, Travailler moins, vivre mieux, Paris, Syros, 1993.

BERGEVIN, Jean-Baptiste, Une réflexion sur le financement d'un revenu garanti, Québec, janvier 2001.

BERNARD, Michel et Michel CHARTRAND, Manifeste pour un revenu de citoyenneté, Montréal, Les éditions du renouveau québécois, 1999.

BLAIS, François, Un revenu garanti pour tous. Introduction aux principes de l'allocation universelle, Montréal, Boréal, 2001.

CASSEN, Bernard, "Faut-il partager l'emploi ? Vers une révolution du travail”, Le Monde diplomatique, mars, 1993.

CASTEL, Robert et als, Chômage : le cas français, rapport au Premier ministre, Commissariat Général au Plan, 1997.

ELLUL, Jacques, Le système technicien, Paris, Calmann-Lévy, 1977.

GORZ, André, Misères du présent, Richesse du futur, Paris, Éditions Galilée,1997.

HUBERMAN, Michaël et Robert LACROIX, Le partage du travail ; une solution au chômage ou un frein à l'emploi, Québec, PUL, 1996.

LAUZON, Léo-Paul, Pour une fiscalité progressiste, juste et équitable, Mémoire présenté à la Commission Séguin sur le déséquilibre fiscal, Montréal, Chaire d'études socio-économiques de l'UQÀM, 2002.

LIPIETZ, Alain, La société en sablier, Le partage contre la déchirure sociale, Paris, Éditions La Découverte, 1996.

MASSOT, Alain, L'école à temps partagé et le partage du travail, Québec, PUL, 1999. 
MORIN, Egard, Les sept savoirs nécessaires à l'éducation du futur, Paris, Éditions du Seuil, 2000.

RAMONET, Ignacio, Le Monde diplomatique, janvier 2000.

RIFKIN, Jeremy, La fin du travail, Paris, Éditions La Découverte, 1996.

ROEDIGER, David et Philip S. FONER, Our Own Time : A History of America Labor and the Working Day, Westport, CT, Greenwood Press, 1989. 


\section{ANNEXES}

$\underline{\text { Retour à la table des matières }}$ 


\section{Tableau I}

Évolution des prestations d'aide sociale au Québec de 1989 à 1996 (Source : BERNARD, Michel et Michel CHARTRAND, Manifeste pour un revenu de citoyenneté, Montréal, Les éditions du renouveau québécois, 1999, p. 78).

$\underline{\text { Retour à la table des matières }}$

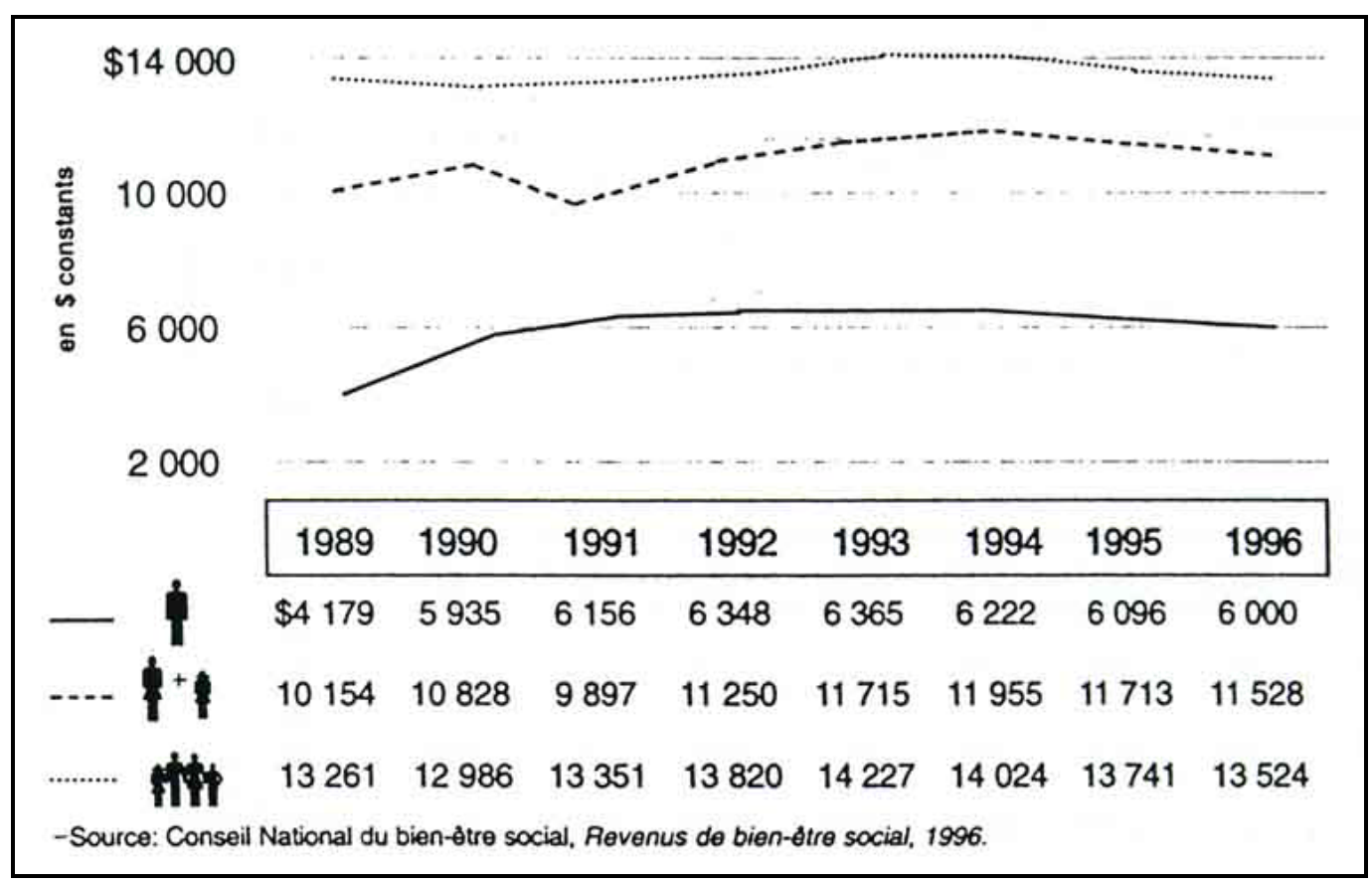




\section{Tableau II}

Évolution du nombre de pauvres au Québec de 1989 à 1996 (Source : BERNARD, Michel et Michel CHARTRAND, Manifeste pour un revenu de citoyenneté, Montréal, Les éditions du renouveau québécois, 1999, p. 78).

$\underline{\text { Retour à la table des matières }}$

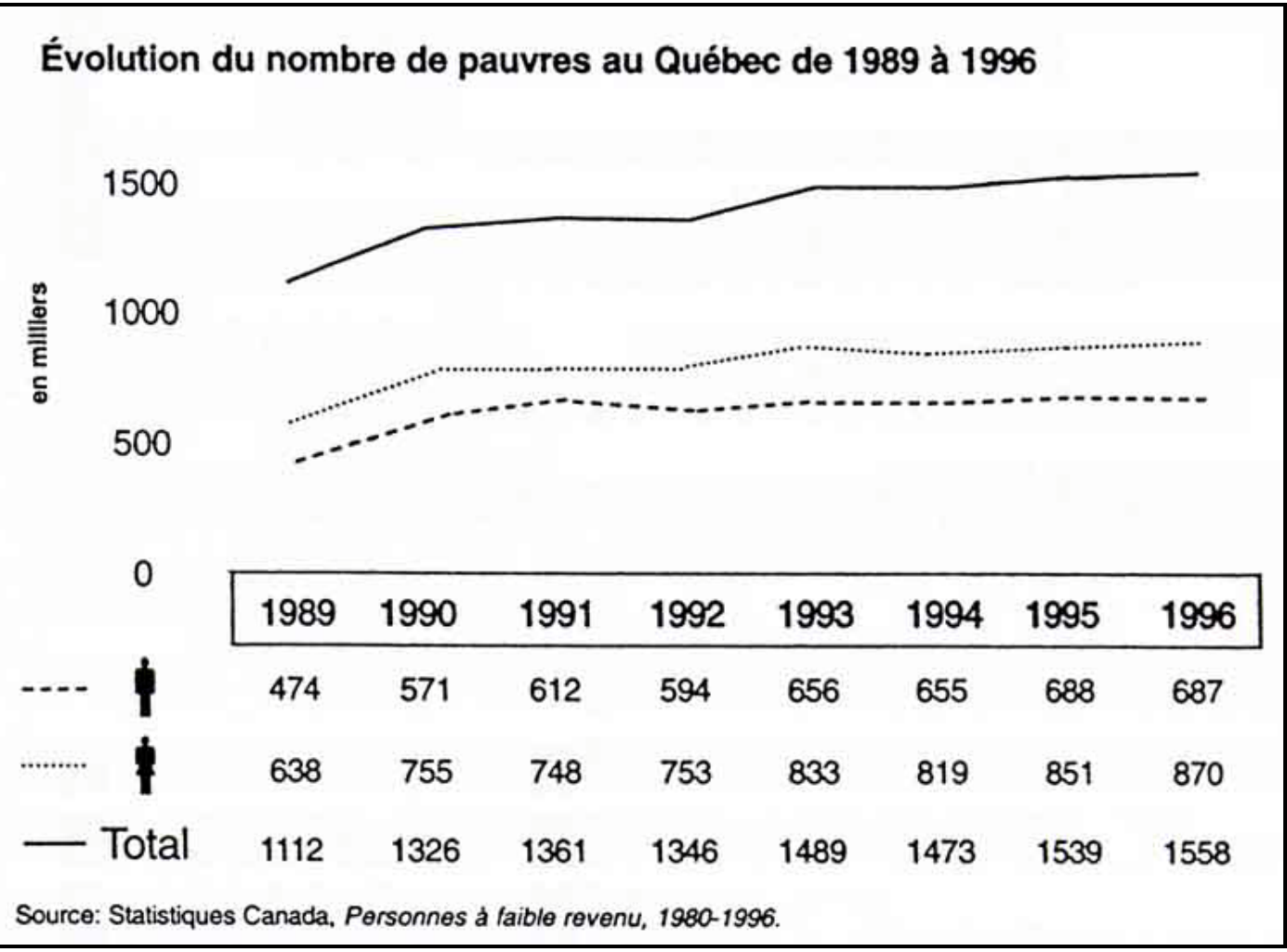




\section{Tableau III}

Principales mesures relevant de la sécurité du revenu (Source: AUBRY, François, L'allocation universelle. Fondements et enjeux, Montréal, CSN, 1999).

$\underline{\text { Retour à la table des matières }}$

\section{Assurance sociale}

Indemnisation des accidents du travail et des maladies professionnelles (Q) * Assurance-emploi (F) *

Régime des rentes $(\mathrm{Q})$ *

Assurance automobile (Q)

Assurance médicaments (Q)

Allocation de maternité $(\mathrm{F})$

\section{Assistance sociale}

Aide aux parents pour leurs revenus de travail (APPORT) (Q) *

Sécurité du revenu (Aide sociale) (Q)

Supplément de revenu garanti $(\mathrm{F})$

Allocation familiale (Q)

Aide financière aux étudiant-es (Q)

Aide financière pour enfants en service de garde (1) (Q)

Allocation pour enfant handicapé (Q)

\section{Mesures universelles}

Pension de la sécurité de la vieillesse et allocation-conjoint-e (F) (2)

\section{Mesures fiscales}

\section{A. Crédits d'impôt remboursables (3)}

Allocation unifiée pour enfants (F)

Frais de garde (1) (Q)

Crédit pour TPS (F)

Crédit pour TVQ (Q)

Remboursement d'impôts fonciers (Q) 
Crédit pour l'hébergement d'un parent (Q)

Crédit remboursable pour frais médicaux (F.Q)

Crédit pour frais d'adoption (Q)

\section{B. Crédits d'impôt non-remboursables et déductions}

Crédit personnel de base $(\mathrm{F}, \mathrm{Q})$

Crédit pour conjoint-e ou équivalent $(F, Q)$

Crédit pour enfant à charge $(\mathrm{Q})$

Crédit pour enfant à charge (4) (Q)

Crédit pour personne déficiente à charge $(\mathrm{F})$

Crédit pour enfant handicapé $(\mathrm{F}, \mathrm{Q})$

Crédit pour aidant naturel (F)

Crédit pour revenu de pension (F)

Crédit en raison d'âge $(F, Q)$

Crédit pour personne vivant seule (Q)

Crédit pour frais médicaux $(\mathrm{F}, \mathrm{Q})$

Crédit pour fonds de travailleurs (F, Q)

Réduction d'impôt à l'égard de la famille (Q)

Frais de scolarité et autres crédits pour études (F, Q)

Déduction pour REER (F, Q) *

Déduction pour fonds de pension agréé $(F, Q)$

Déduction pour frais de garde $(\mathrm{F})$ *

Déduction pour frais de préposé-e aux soins (F)

F = fédéral; Q = Québec

* Mesures liées à la participation à une activité de travail

(1) Mesure appelée à disparaître avec l'introduction des places à 5 \$ en garderie

(2) Officiellement toujours une mesure universelle mais soumise à un régime spécial d'imposition

(3) Ces mesures fiscales sont de nature « assistance sociale » car elles ne sont versées qu'aux personnes à faible revenu

(4) Pour les familles monoparentales 


\section{Tableau IV}

Financement du projet d'un revenu garanti, (Source: BERGEVIN, JeanBaptiste, Une réflexion sur le financement d'un revenu garanti, Québec, janvier 2001, p. 14).

$\underline{\text { Retour à la table des matières }}$

ESTIMATION DU COÛT BRUT DU PROJET REVENU GARANTI (voir le tableau \#3A col.3)

DIVERSES RÉCUPÉRATIONS À PARTIR DES BUDGETS

EN PLACE

- Récupération du Revenu Garanti payé à 53,352 des déclarants dont les revenus sont tels qu'ils doivent retourner le tout ou partie du RG reçu (voir Tableau \#3a)

- Récupération de certaines dépenses fiscales consenties aux particuliers (voir Tableau \#2 et portion 'A' de l'Annexe A)

- Récupération de certaines dépenses fiscales consenties aux sociétés (voir Tableau \#2 et portion 'B' de l'Annexe A)

- Conversion des prestations du Régime de 17,855 pensions canadien en prestation du Revenu Garanti

- Conversion des programmes provinciaux de prestations et allocations familiales 


\section{Tableau V}

Hypothèse d'un revenu garanti de 7,500\$ par adulte, perçue, dans le contexte d'autres données de sécurité du revenu en \% de 1999, (Source: BERGEVIN, Jean-Baptiste, Une réflexion sur le financement d'un revenu garanti, Québec, janvier 2001, p. 18).

$\underline{\text { Retour à la table des matières }}$

\begin{tabular}{lccc}
\hline Statut familial & $\begin{array}{l}\text { Système actuel } \\
\text { (Québec) }(1)\end{array}$ & $\begin{array}{l}\text { Présent modèle } \\
\$ 7,500 / \text { adulte } \\
(2)\end{array}$ & $\begin{array}{l}\text { Seuil faible } \\
\text { revenu Statisti- } \\
\text { que Canada (3) }\end{array}$ \\
\hline Personne seule & $9024 \$$ & $7500 \$$ & $15355 \$$ \\
Couple sans enfant & $10030 \$$ & $11506 \$$ & $19194 \$$ \\
Monoparentale, 1 enfant & $12530 \$$ & $14006 \$$ & $23872 \$$ \\
Monoparentale, 2 enfants & $11958 \$$ & $17706 \$$ & $23872 \$$ \\
Couple + 1 enfant & $13833 \$$ & $19581 \$$ & $28895 \$$ \\
\hline
\end{tabular}

(1) Il s'agit ici des prestations et allocations familiales versées (modèle Québec).

(2) Il s'agit de l'hypothèse retenue dans notre modèle comme Revenu Garanti de base, à laquelle sont ajoutées les allocations familiales et les prestations familiales versées selon le modèle de Québec.

(3) Il s'agit du seuil de faible revenu établi par Statistique Canada pour 1996 et indexé de 4.5\% pour 1999 (sigle anglais de ce faible revenu : " low income cut-off » ou LICO). Ce seuil n'a jamais été appliqué. 


\section{Tableau VI}

Montant des impôts reportés au bilan des compagnies, fin de l'année 1999, (Source : LAUZON, Léo-Paul, Pour une fiscalité progressiste, juste et équitable, Mémoire présenté à la Commission Séguin sur le déséquilibre fiscal, Montréal, Chaire d'études socio-économiques de l'UQÀM, 2002, p. 92)

$\underline{\text { Retour à la table des matières }}$

\begin{tabular}{lcc}
\hline Catégories & $\begin{array}{c}\text { Nombre } \\
\text { d'entreprises }\end{array}$ & $\begin{array}{c}\text { Montants d'impôts } \\
\text { reportés }\end{array}$ \\
\hline Impôts reportés créditeurs & 272 & 34745,8 \\
Impôts reportés débiteurs & 80 & $(4894,3)$ \\
Impôts reportés nuls & 38 & 0,0 \\
\hline TOTAL & 390 & 29851,5 \\
\hline
\end{tabular}




\section{Schéma I}

Comparaison entre l'impôt négatif et l'allocation universelle du point de vue de leur structure distributive (Source : BLAIS, François, Un revenu garanti pour tous. Introduction aux principes de l'allocation universelle, Montréal, Boréal, 2001).

\section{$\underline{\text { Retour à la table des matières }}$}

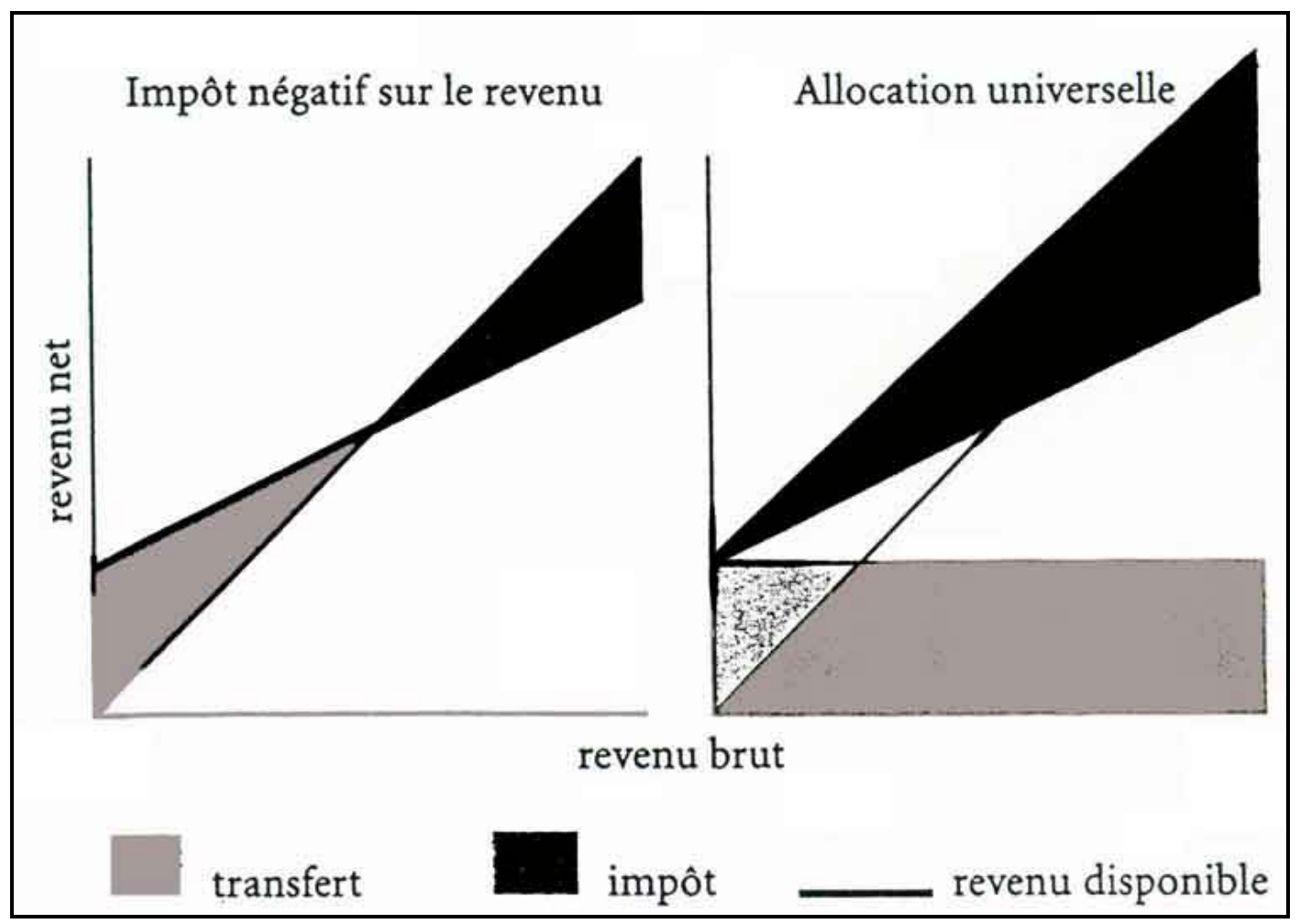

\section{Fin du texte}

